\title{
Krafttraining vor totalem Hüftgelenkersatz
}

Holsgaard-Larsen A et al. Effects of progressive resistance training prior to total HIP arthroplasty - a secondary analysis of a randomized controlled trial. Osteoarthritis Cartilage 2020; 28: 1038-1045. doi: 10.1016/j.joca.2020.04.010

Ein Teil der Patienten mit einer schweren Hüftgelenkarthrose leidet auch nach einem totalen Gelenkersatz unter anhaltenden körperlichen Einschränkungen und muskulären Defiziten. Lässt sich durch ein präoperatives Krafttraining die funktionelle Leistungsfähigkeit der Patienten verbessern? Und wie wirkt sich ein solches Training auf die Schmerzbelastung, die Aktivität, die gelenkbezogene Lebensqualität sowie die Muskelkraft aus?

Diesen und anderen Fragen gingen dänische Wissenschaftler im Rahmen einer randomisierten kontrollierten Studie nach. An der Untersuchung nahmen 80 Patienten teil, welche sich aufgrund einer primären Coxarthrose an der Universitätsklinik Kopenhagen einer totalen Hüftarthroplastik unterzogen. Die Hälfte der Studienteilnehmer absolvierte vor dem Eingriff über einen Zeitraum von 10 Wochen zweimal pro Woche ein auf die Explosivkraft der unteren Extremität abgestimmtes Krafttraining unter physiotherapeutischer Anleitung. Die 40 Kontrollen erhielten die übliche Operationsvorbereitung, welche unter anderem eine Anleitung zu gering intensivem Heimtraining ohne spezifische Kraftübungen umfasste. Als primären Studienendpunkt definierten die Wissenschaftler das mittels Hip Osteoarthritis Outcome Score (HOOS) erfasste subjektive Behandlungsergebnis der Subskala „Aktivitäten des täglichen Lebens" nach 12 Monaten. Ferner objektivierten sie die Subskalen „Schmerz“, „Symptome“, „Sport und Freizeit" sowie "gelenkbezogene Lebensqualität" nach 3, 6, 9 und 12 Monaten Nachbeobachtungszeit. Zusätzlich erhoben die Forscher verschiedene objektive funktionelle Parameter wie die Gehgeschwindigkeit, das Treppensteigen, das Aufstehen aus dem Sitzen sowie die isometrische Muskelkraft der Hüft- und Knieextensoren und die Körperzusammensetzung. 


\section{Ergebnisse}

Das Studienkollektiv bildeten 52 Frauen und 28 Männer im Durchschnittsalter von 70,4 Jahren. Nach der zwölfmonatigen Nachbeobachtungszeit konnten Daten von je 34 Patienten beider Studiengruppen ausgewertet werden. Nach 12 Monaten erwies sich das präoperative Krafttraining weder im Hinblick auf die Aktivitäten des täglichen Lebens noch im Hinblick auf die weiteren HOOS-Subskalen als vorteilhaft. Gleiches galt für das Aufstehen aus dem Sitzen, die Gehgeschwindigkeit sowie die fettfreie Körpermasse. Lediglich im Hinblick auf das Hinauf- und das Hinuntersteigen von Treppen beobachteten die Forscher in der Interventionsgruppe einen signifikanten Effekt von 1,3 bzw. 1,6 Sekunden. Zum Erhebungszeitpunkt nach 3 Monaten zeigte sich ferner ein klinisch signifikanter Behandlungsvorteil des Krafttrainings im Hinblick auf die HOOS-Subskala „Sport und Freizeit“, die Muskelkraft der Knieextensoren an beiden Beinen, das Aufstehen aus dem Sitzen sowie die maximale Gehgeschwindigkeit und das Treppensteigen.

\section{FAZIT}

Ein Training der Explosivkraft vor totalem Hüftgelenkersatz, so das Fazit der Autoren, wirkt sich 12 Monate nach der Operation im Vergleich zur Routinebetreuung weder günstig auf die funktionelle Leistungsfähigkeit noch auf die Muskelkraft der Patienten aus. Allerdings bietet das Krafttraining nach 3 Monaten einen Rehabilitationsvorteil, so dass ihrer Ansicht nach eine entsprechende präoperative Vorbereitung unter dem Aspekt der beschleunigten postoperativen Erholung in Betracht gezogen werden sollte.

Dr. med. Judith Lorenz, Künzell 